\title{
Embedded Fiber-Optic Sensing for Accurate Internal Monitoring of Cell State in Advanced Battery Management Systems
}

\section{Part 2: Internal Cell Signals and Utility for State Estimation}

\author{
Anurag Ganguli ${ }^{1}$, Bhaskar Saha ${ }^{1}$, Ajay Raghavan ${ }^{1 *}$, Peter Kiesel ${ }^{1}$, Kyle Arakaki ${ }^{1}$, Andreas \\ Schuh $^{1}$, Julian Schwartz ${ }^{1}$, Alex Hegyi ${ }^{1}$, Lars Wilko Sommer ${ }^{1}$, Alexander Lochbaum ${ }^{1}$, Saroj \\ Sahu ${ }^{1}$, and Mohamed Alamgir ${ }^{2}$ \\ ${ }^{1}$ Palo Alto Research Center (PARC, a Xerox Company), Palo Alto, CA 94304, U.S.A. \\ ${ }^{2}$ LG Chem Power, Troy, MI 48083, U.S.A.
}

\begin{abstract}
A key challenge hindering the mass adoption of Lithium-ion and other next-gen chemistries in advanced battery applications such as hybrid/electric vehicles (xEVs) has been management of their functional performance for more effective battery utilization and control over their life. Contemporary battery management systems (BMS) reliant on monitoring external parameters such as voltage and current to ensure safe battery operation with the required performance usually result in overdesign and inefficient use of capacity. More informative embedded sensors are desirable for internal cell state monitoring, which could provide accurate state-of-charge (SOC) and state-of-health ( $\mathrm{SOH})$ estimates and early failure indicators. Here we present a promising new embedded sensing option developed by our team for cell monitoring, fiber-optic sensors. High-performance large-format pouch cells with embedded fiber-optic sensors were fabricated. This second part of the paper focuses on the internal signals obtained from these FO
\end{abstract}

\footnotetext{
*Corresponding author. Email: raghavan@parc.com; Phone: 1-650-812-4724; Fax: 1-650-812-4334
} 
sensors. The details of the method to isolate intercalation strain and temperature signals are discussed. Data collected under various xEV operational conditions are presented. An algorithm employing dynamic time warping and Kalman filtering was used to estimate state-of-charge with high accuracy from these internal FO signals. Their utility for high-accuracy, predictive state-ofhealth estimation is also explored.

\section{Introduction}

As explained in part 1 of this two-part paper, a better understanding and real-time monitoring of internal cell state with accurate sensors is of critical need for effective control by battery management systems (BMS). This can play a key role in accelerating adoption of Lithium (Li)-ion batteries for clean energy technologies [1]-[3]. BMS for Li-ion cells perform a variety of functions such as cell balancing, state-of-charge (SOC), state-of-health ( $\mathrm{SOH})$ and state-of-power (SOP) estimation, failure prevention, and battery protection. BMS today typically monitor parameters such as voltage, current, and temperature externally to estimate cell state parameters. Common contemporary methods include Coulomb counting, open circuit voltage (OCV)-based approaches, and dynamical model-based approaches.

In Coulomb counting, the current is integrated over time and divided by the battery capacity to estimate SOC. This can be challenged in fielded packs as the low-cost current sensors typically used there have measurement drift errors that accumulate over time. The uncertainty in initial SOC and changing battery capacity over time can further complicate this method. In [4], an enhanced Coulomb counting method for SOC estimation was presented that uses derived empirical relationships between initial SOC, voltage and current, and dynamically re-calibrating the battery capacity. Using this approach, the maximum SOC estimation error was approximately 
$3 \%$. It is not clear, however, whether that level of accuracy holds for more aggressive xEVrelevant battery loading profiles.

OCV can be computed by letting the battery rest for a sufficient duration of time ([5][7]). SOC can then be estimated using the pre-calibrated SOC-OCV relationship for that cell chemistry (or obtained using a very slow constant current charge). However, this method is of limited utility in real-world applications with real-time estimation requirements.

The dynamical model-based approach can broadly be classified into equivalent electrical circuit modeling and electrochemical modeling. The equivalent electrical circuit modeling approach is by far the most popular. There is a large body of research in this area. In [8], the authors employ an impedance spectroscopy approach to develop a non-linear electric-circuit model of an absorbent glass mat lead-acid battery. In [9], the authors evaluate and compare different electric circuit equivalent models of lithium-ion battery. According to their studies, the dual-polarization model, i.e. modeling independently the concentration and electrochemical polarizations, is the most accurate in estimating SOC.

In [10], the authors use a reduced order model of a Li-ion battery in conjunction with an Extended Kalman Filter (EKF) to estimate the SOC. To account for model errors, the measurement noise covariance in the EKF is modified based on the estimated SOC, the current, and the dynamics of diffusion, charge transfer and double layer. In [5], the authors address the problem that in practice there is some variation in the OCV versus SOC relationship from one Li-ion cell to the other. A new definition of capacity is proposed to minimize the variation across different cells. This leads to different capacities across different cells. A dual EKF is implemented that simultaneously estimates both capacity and SOC. As in [10], the measurement noise covariance is adaptively changed to account for model errors. 
Electrochemical models attempt to build the model from first principles. Examples of these models include the pseudo two-dimensional model, the single particle model and the porous electrode model [11]. With such models, a trade-off exists between model realism and solution time. In [12], the authors develop a partial differential equation-based observer using the back-stepping control approach.

In part 1 of this paper, we made the case for direct internal monitoring with more informative embedded sensors to provide accurate SOC estimates and early indications of incipient failure for BMS. We presented fiber optic (FO) sensors as attractive candidates for embedding as sensors in Li-ion and other advanced batteries. The successful fabrication of highperformance large-format Li-ion pouch cells with embedded FO sensors (referred to as "FOcells" in this paper) and assembly into commercial xEV modules by our team were discussed in part 1 of this paper. This second part of the paper focuses on the internal strain and temperature signals obtained from these fiber-optic sensors under various xEV operational conditions and their utility for high-accuracy SOC and SOH estimation algorithms.

The contributions and organization of this part of the two-part paper is as follows. In Section 2, we describe the various parameters that can be sensed or derived using FO sensing. These parameters include temperature, strain and current. We describe a novel computational approach for strain-temperature separation and experimentally validate the approach. In Section 3, we explain the relationship of strain with SOC in cell- and module-level tests and present our SOC estimation algorithm. The SOC estimation is based on a combination of FO based strain sensing and Coulomb counting. In Section 4, we describe the impact of aging on the strain-SOC relationship and our $\mathrm{SOH}$ estimation algorithm. In particular, we use measured strain to estimate the capacity of the battery and also predict the capacity up to 10 cycles into the future. Finally, 
we conclude with a summary and some thoughts for future research directions building on this work.

\section{Sensing Parameters of Interest and Strain-Temperature Separation}

The details of the large-format FO-cells fabricated by our team were presented in part 1 of this paper. It is shown again here in Figure 1 for reference. As mentioned and illustrated there, the FO cable within the cell shown there includes two elements of a particular class of FO sensors, fiber Bragg grating (FBG) sensors [13]. The FBG sensors are sensitive to strain and temperature, measured by monitoring their reflected wavelength shifts $\Delta \lambda$. The nominal cell capacity is approximately $15 \mathrm{Ah}$. The cell's anode material is graphite and cathode material is a blend of nickel-manganese oxide and manganese spinel.

It should be mentioned that while the FBG is monitoring the electrode around a single point, intuitively strain at a point in the electrode is not only affected by local SOC but also by expansion/contraction from lithiation/delithiation at other points in the electrode layer (since they are all mechanically part of the same electrode structure). Therefore, the strain measured at one point is expected to be indicative of SOC over a much larger area of the electrode rather than just the local SOC at that point.

One of the two FBG sensors was enclosed in a special tubing to allow it to slide freely and thereby make it selectively sensitive to thermal strain alone. The measured wavelength shift

of the "reference" FBG sensor in the tubing is then subtracted from the wavelength shift of the adjacent FBG sensor sensitive to total electrode strain so that temperature variations are compensated. The compensation method is described next.

The reference FBG in the tubing, referred to herein as "loose" FBG, measures only the local temperature change $\Delta T[14]$ : 


\section{[Deleted Figure 1(a)]}

(a)

\section{[Deleted Figure 1(b)]}

(b)

Figure 1: (a) Picture of large format pouch sealed successfully with FO cable; (b) Zoomed-in view of the pouch region near the FO cable. (a) Functional configuration of FO cable with fiber Bragg grating sensors in large format xEV pouch cells (b) Photograph of FBG sensor pair attached to the large format cell electrode before the cell was sealed and activated.

$$
\Delta \lambda_{\text {loose }}=\bar{K}_{T} \Delta T
$$

The adjacent FBG (referred to herein as "fixed") measures a combination of strain $\epsilon$ and temperature change [14]:

$$
\Delta \lambda_{\text {fixed }}=K_{\epsilon} \epsilon+K_{T} \Delta T=\bar{\epsilon}+K_{T} \Delta T
$$

Equations (1) and (2) can be rewritten as follows:

$$
\Delta \lambda_{\text {fixed }}=\bar{\epsilon}+K_{T}^{*} \Delta \lambda_{\text {loose }}
$$

We can recover the modified strain, $\bar{\epsilon}$, if the constant $K_{T}^{*}$ can be estimated. We refer to this constant as the "temperature compensation factor". This factor can be estimated in two ways.

Experimental approach to determining the temperature compensation factor

In this approach, a cell at rest is first heated to a certain temperature. The values of $\Delta \lambda_{\text {fixed }}$ and $\Delta \lambda_{\text {loose }}$ are recorded. Because the cell is at rest, the value of $\bar{\epsilon}$ is zero. Therefore, the steady state ratio of $\Delta \lambda_{\text {fixed }}$ to $\Delta \lambda_{\text {loose }}$ gives the value of $K_{T}^{*}$.

\section{Computational approach to determining the temperature compensation factor}

This approach is based on the hypothesis that the modified strain as a function of SOC is invariant to the rate of charge (or discharge). In this approach, the cell is charged and discharged 
multiple times under constant current conditions. It is ideal if the charge and discharge currents are different for the different cycles. The procedure is described below. In this procedure, $N$ is the number of times the cell is charged and discharged; $I^{j}(t)$ refers to the current at time $t$ during the $j^{t h}$ cycle. Likewise, let $\Delta \lambda_{\text {loose }}^{j}(t)$ and $\Delta \lambda_{\text {fixed }}^{j}(t)$ denote the measured wavelength shifts from the two FBG sensors at time $t$ during the $j^{\text {th }}$ cycle.

1. For each cycle $j$, compute the state of charge at each time instant $S O C^{j}(t)$

2. For each cycle $j$, compute the time instant when the SOC is 1 percent. Denote this by $t_{1}^{j}$.

3. Repeat the previous step for SOC equal to 2 percent all the way to 100 percent in increments of the 1 percent. Let $t_{X}^{j}$ denote the time instant when the SOC is $X$ percent in the $j^{\text {th }}$ cycle.

4. For a given temperature compensation factor $K$ and $\operatorname{SOC} X$, let:

$$
\mu(K, X)=\frac{1}{N} \sum_{\{j=1\}}^{\{N\}}\left(\Delta \lambda_{\text {fixed }}^{j}\left(t_{X}^{j}\right)-K \Delta \lambda_{\text {loose }}^{j}\left(t_{X}^{j}\right)\right)
$$

5. Let:

$$
\sigma(K, X)=\frac{1}{N} \sum_{\{j=1\}}^{\{N\}}\left(\Delta \lambda_{\text {fixed }}^{j}\left(t_{X}^{j}\right)-K \Delta \lambda_{\text {loose }}^{j}\left(t_{X}^{j}\right)-\mu(K, X)\right)^{2}
$$

6. Let:

$$
J(K)=\sum_{X=1}^{100} \sigma(K, X)
$$

7. Finally the compensation factor is derived by the equation:

$$
K_{T}^{*}=\operatorname{argmin}_{K} J(K)
$$


Figure 1 illustrates the concept behind the computational approach. Both the above methods can be used to determine the factor during the cell manufacturing process itself, particularly during the activation step of the cell where it is subjected to aging under controlled thermal conditions.
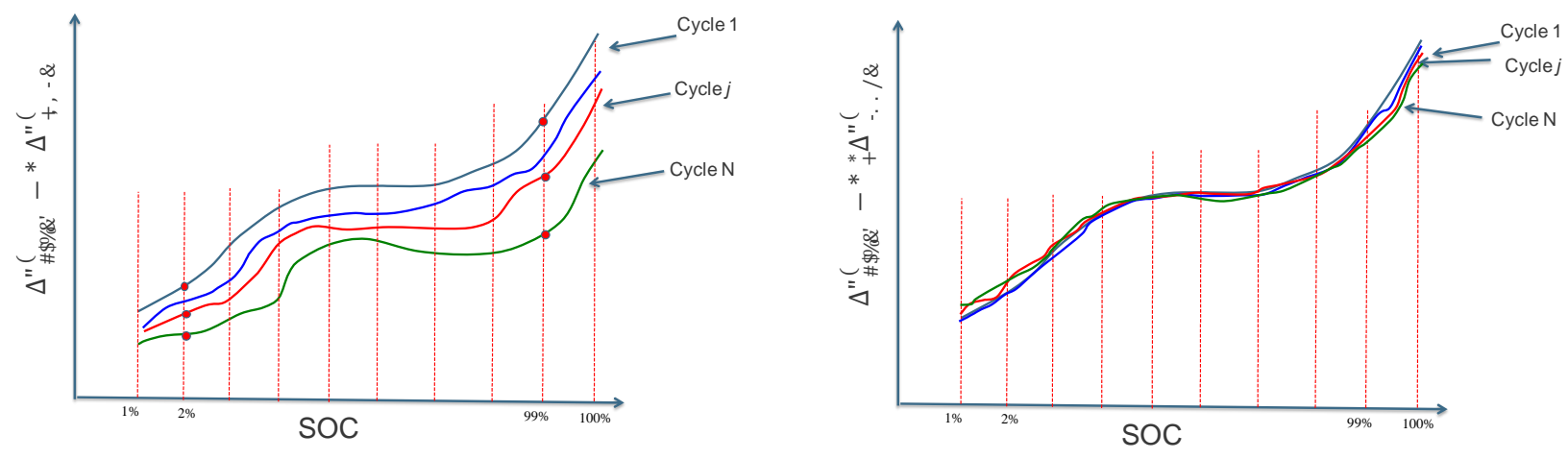

Figure 1: Estimation of the optimal compensation factor for strain-temperature separation in the FBG signal. The strain versus SOC curves are the closest to one another when the temperature compensation factor is the optimal.

Comparison of experimental and computational approaches

We conducted two sets of standard charge and discharge experiments (referred to as Experiment 1 and Experiment 2) and determined the temperature compensation factors according to computational method described above. The temperature compensation factor determined was 4.1 and 3.7 respectively.

Independently, we determined the temperature compensation factor using the experimental approach. Note that for small temperature changes and as the thermal chamber ramps up, the wavelength shifts for the strain and temperature signals are small and therefore the ratio of $\Delta \lambda_{\text {fixed }}$ to $\Delta \lambda_{\text {loose }}$ is prone to numerical noise. Over the steady state (as the thermal chamber equilibrates after 1 hour) and at larger values of wavelength shift of the loose FBG (approximately $>20 \mathrm{pm}$ ), the ratio is constant. The temperature factor determined over the 
region where the ratio is constant is 3.88 . The difference between the experimental and the computational approaches is less than $5 \%$.

It should be noted that FBG sensors could also be used for current sensing [13]. However, since that is already well established in the literature, we chose not to explore that further within this study. For our present experiments reported here, we had ready access to cell current value from the battery cycler. For the eventual deployable version of our technology in fielded battery management systems, it is possible to extend our FO multiplexed sensing capabilities to monitor pack current as well.

\section{Relating Electrode Strain to SOC}

The functional principle of Li-ion cells is based on lithium intercalation. The insertion/release of Li ions into/from the electrode active materials is associated with structural changes of the electrode active materials and consequently with volume change. For most Li-ion chemistries, the cell volume change is predominantly caused by the anode material. Thus, the battery expands during charge and contracts during discharge [15]-[17]. The anode expands during charging as Li ions intercalate into the electrode. During discharging, the Li ions deintercalate from the anode leading to a contraction. The cathode expands during discharging as it accepts the Li ions back. The cathode expansion, however, is usually much smaller than the anode [15].

As mentioned in part 1 of this 2-part manuscript, the strain FBG is attached to the electrode using styrene-butadiene-rubber, a binder confirmed to survive over the life of the cell within the Lithium-ion cell environment for holding the anode particles together reliably. Accordingly, the FBG signal is expected to be reliably representative of electrode strain over time. 
Initially, cell-level characterization tests were done over static cycles at different $\mathrm{C}$ rates at $20^{\circ} \mathrm{C}$ (room temperature). It should be noted that the cells take 10-20 "break-in" chargedischarge cycles for the SEI layer to settle and the strain signals to become repeatable. Following the "break-in" standard cycles, the cells were charged and discharged using a range of currents from a rather low value $(\mathrm{C} / 10)$ to a value as high as $5 \mathrm{C} / 3$. The cell was charged to the upper voltage limit of $4.15 \mathrm{~V}$ using the respective constant current and then charged potentiostatically until the cutoff current was reached. Thereafter, after a 1-hour rest period, the cell was discharged at the corresponding current to the cutoff voltage of $2.5 \mathrm{~V}$.

For the initial cell-level characterization results reported here, the large-format FO-cells were placed in the module, but cycled individually one at a time (leaving all other cells in the module electrically idle for cycling a particular cell). Coolant was cycled through the active thermal management system and subjected to various charge-discharge rates. The results from these initial tests are shown in Figure 2(a). As seen there, the static cycle test results showed promise with repeatable curves across the different $\mathrm{C}$ rates spanning $\mathrm{C} / 10$ through $5 \mathrm{C} / 3$. It should be noted that the strain curves corresponding to the $\mathrm{C}$ rates are only shown from $0 \%$ SOC through the limit of the constant current phase. Beyond the constant current phase, over the constant voltage phase through the potentiostatic charge phase, the strain curves would not be representative of the higher $\mathrm{C}$ rates and therefore were not included.

This spread in the strain curves at different $\mathrm{C}$ rates is much smaller than the comparable spread for an external sensing configuration, where the fiber-optic sensors were bonded on the cell skin surface at a similar central location to monitor strain and temperature, as reported earlier by our team [18]. This can be attributed to the much better thermal robustness and better temperature compensation for the strain FO sensor in the internal sensing configuration. In this 
configuration, the FO sensor is attached to a graphitic anode with a much smaller thermal expansion coefficient and is less sensitive to temperature changes. Furthermore, the reference temperature sensor directly senses the higher electrode temperature at the high $\mathrm{C}$ rates.

In contrast, for the external configuration the FO sensor is attached to the metallic skin of the pouch cell that has a higher thermal expansion coefficient and is thus more sensitive to temperature changes at higher $\mathrm{C}$ rates and dynamic oscillations. Furthermore, the external FO temperature sensor is insulated from the true internal electrode temperature by the separator layers. At the highest C-rates (and aggressive dynamic cycles), this temperature differential can be considerable and can contribute to differences in the recovered strain curves at lower $\mathrm{C}$ rates where the temperature differentials are milder.

Following the cycling tests at $20^{\circ} \mathrm{C}$, tests were done placed under hot $\left(45^{\circ} \mathrm{C}\right)$ and cold ($15^{\circ} \mathrm{C}$ ) conditions in temperature-controlled chambers. The curves continued to show similar shapes at $45^{\circ} \mathrm{C}$, as shown in Figure 2(b). This is also true even in the more extreme cold condition where the cell was charged/discharged at the LG Chem-suggested maximum C rates at $-15^{\circ} \mathrm{C}$, as shown in Figure 2(c). 

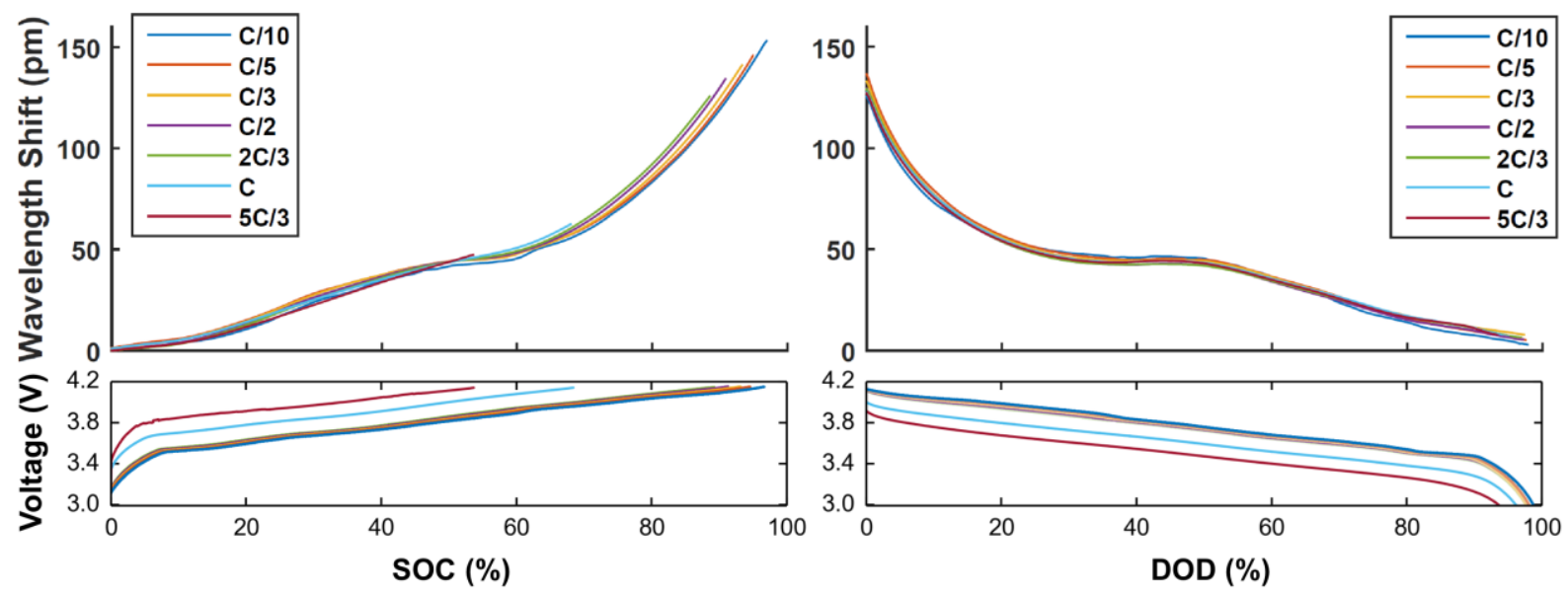

(a)
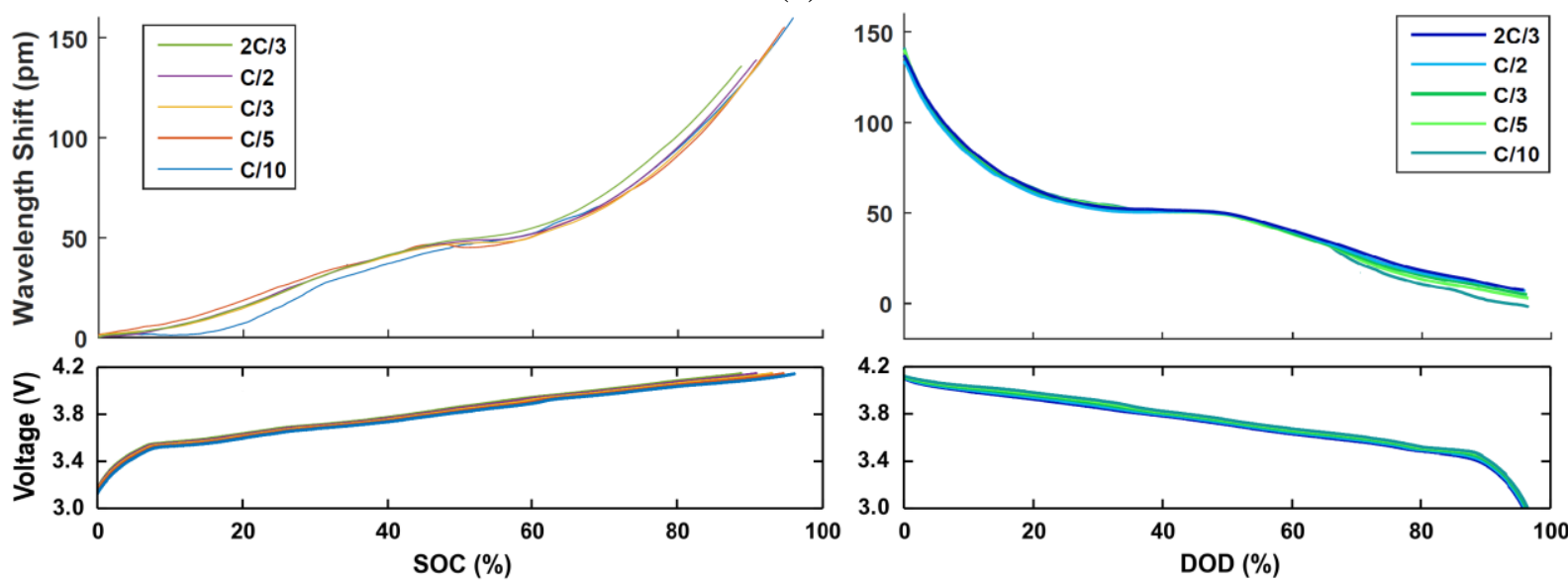

(b)
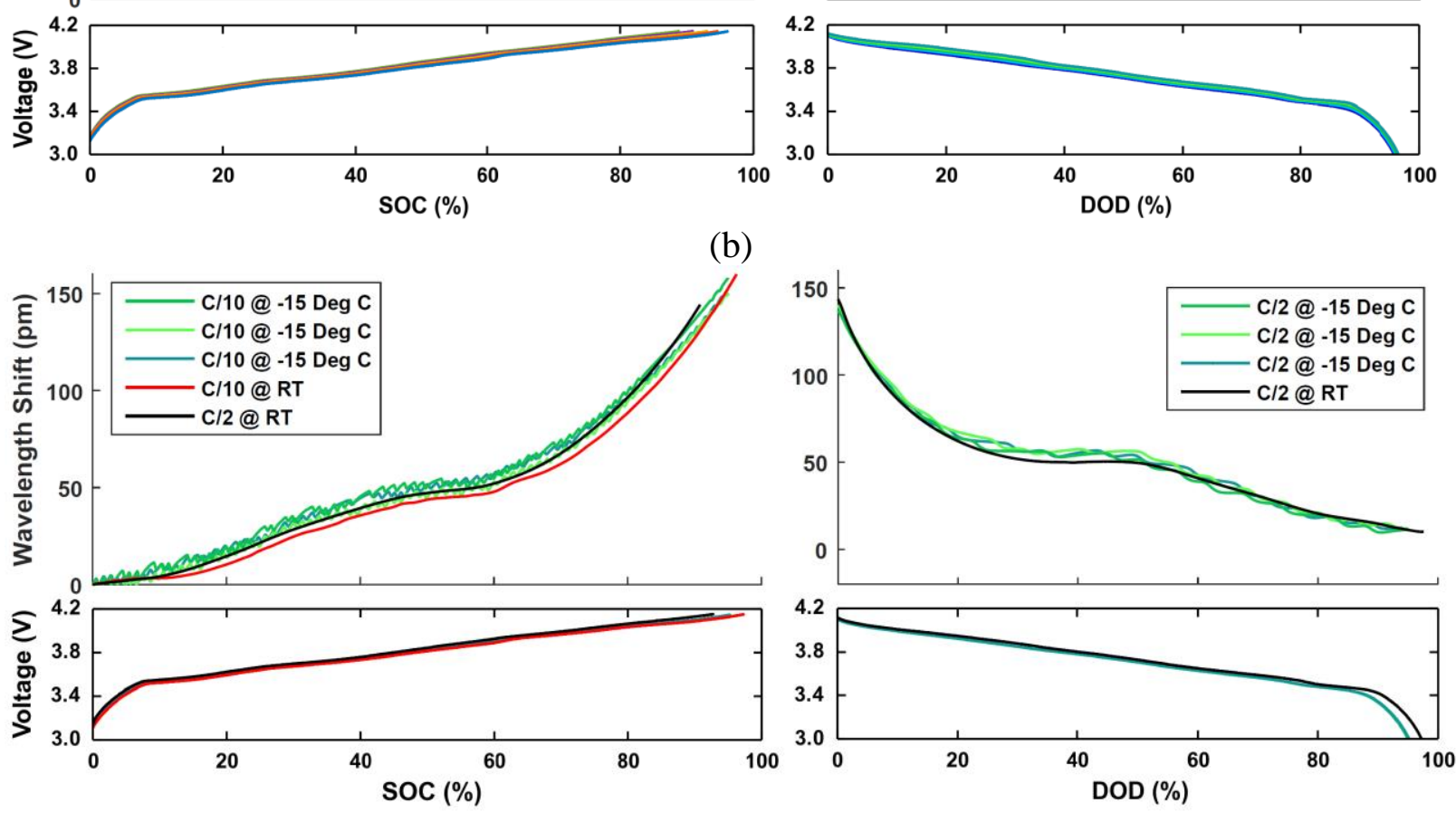

(c)

Figure 2: Strain curves from internal sensors in large-format cells places in four-cell module in initial static cycle cell-level tests at various $\mathrm{C}$ rates and temperatures: (a) from $\mathrm{C} / 10$ thru $5 \mathrm{C} / 3$ at (a) $25^{\circ} \mathrm{C}$, (b) from $\mathrm{C} / 10$ thru $2 \mathrm{C} / 3$ at $45^{\circ} \mathrm{C}$, and (c) $\mathrm{C} / 10$ at $-15^{\circ} \mathrm{C}$ with $\mathrm{C} / 10, \mathrm{C} / 2$ at $25^{\circ} \mathrm{C}$. 
Subsequently, following the above cell-level tests, the cells in the 4-cell module (as described in part 1 of this 2-part manuscript) were electrically coupled together by connecting them in parallel to simulate the fielded module use case. The cells were then cycled with another set of static cycles comprising of charge rates at $\mathrm{C} / 2$ and discharge rates ranging from $\mathrm{C} / 5$ to $\mathrm{C}$ at different temperatures. The shape similarity of the recovered electrode strain curves, shown in Figure 3, allows for the use of machine learning algorithms such as dynamic time warping [19] for SOC estimation (Figure 4). These allow for training with a minimal data set. Note, however, that because these methods work by matching patterns, they rely on the availability of a sufficient amount of time history of the data for the method to work effectively. The algorithm yielded average SOC estimation accuracy of better than $1 \%$ SOC, as shown in Table 1, with the worst-case error also being below $1 \%$.

After the static cycles were done on the FO-cells, the module (with cells all electrically connected in parallel) was subjected to a series of rapid xEV dynamic cycles, following the US06 [20] and UDDS [21] drive profiles (scaled down accordingly for the battery here) with 1shold times. The recovered strain signals from this test are shown in Figure 5 (a).

The dynamic cycle signals are shown in red and overlaid on the static cycle signals in blue. The general agreement between the two data sets indicates that the static cycle data should suffice as training set to predict SOC for the dynamic cycles as test sets. There exist, however, certain regions over which the agreement is less. Due to this reason, the approach based on dynamic time warping performed inadequately in terms of SOC accuracy. We then explored an alternate approach described next.

A semi-empirical model was developed for calculating the state-of-charge (SOC) from sensor output. The model is explained below: 


$$
\begin{gathered}
q(t+\Delta T)=q(t)+K(t) i(t) \Delta T / C+w_{q}(t) \\
K(t+\Delta T)=K(t)+w_{K}(t) \\
\mu(t+\Delta T)=h(q(t+\Delta T))+v_{\mu}(t+\Delta T)
\end{gathered}
$$
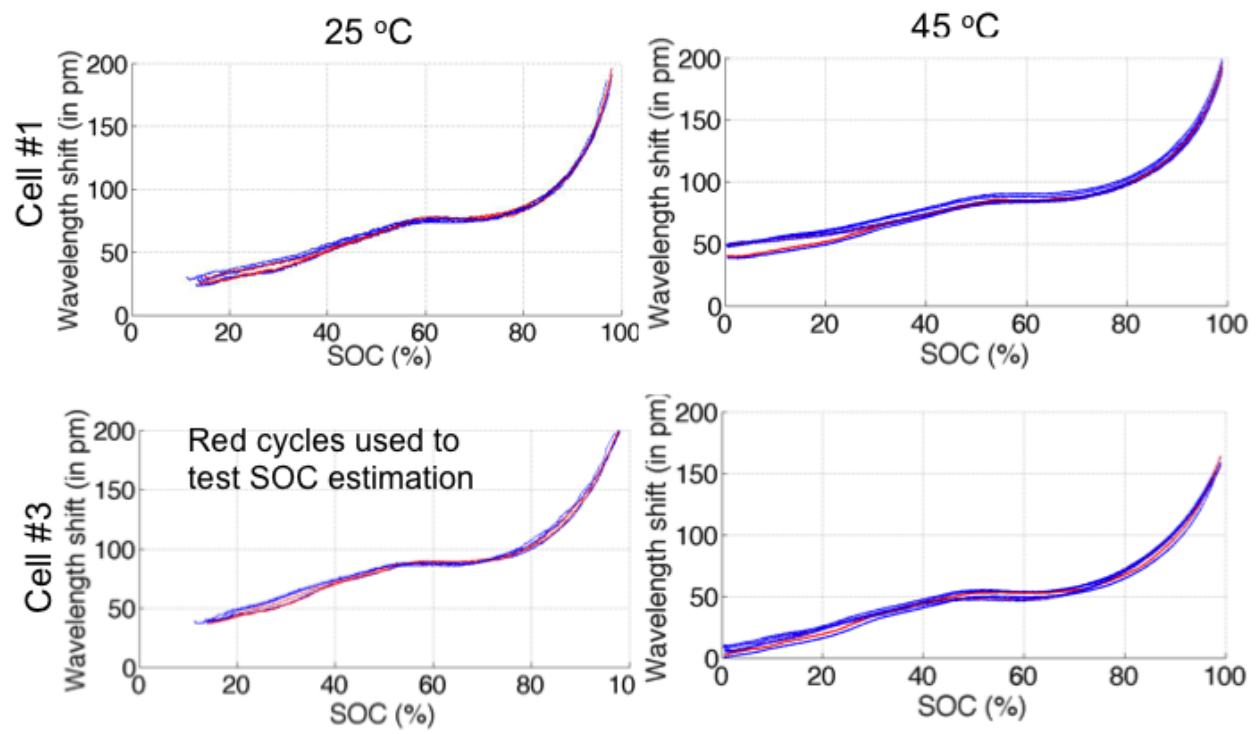

Figure 3: Internal electrode strain signals from the large format FO-cells for cycles comprising of charge rates at $\mathrm{C} / 2$ and discharge rates from $\mathrm{C} / 5$ to $\mathrm{C}$ at two different temperatures.

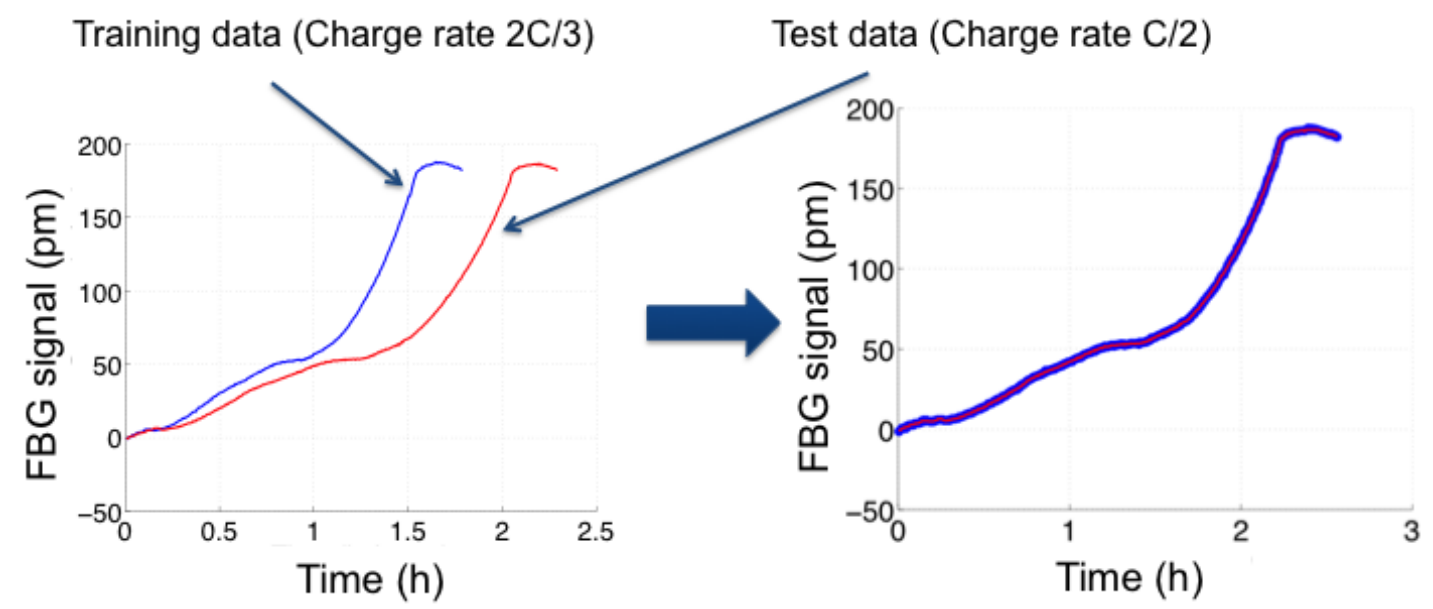

Figure 4: Dynamic time warping approach used for SOC estimation. 


\section{Mean error}

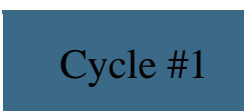

Cycle \#2

Cell \#1

$0.34 \%$

$0.24 \%$

Cell \#3

$0.08 \%$

$0.16 \%$

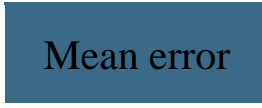

Cycle \#1

Cycle \#2

Cell \#1

$0.71 \%$

$0.93 \%$

Cell \#3

$0.72 \%$

$0.96 \%$

Table 1: SOC estimation results based on dynamic time warping for static cycles using the internal electrode signal from the large-format FO-cells (internal sensing)

In the above model, $q(t)$ denotes the SOC at time instant $t$. The cell capacity is denoted by $C$. The instantaneous current is denoted by $i$. The parameter $K$ signifies the fraction of input charge or discharge that contributes towards the SOC. Ideally, $K(t)$ should be equal to one for all times. The observed temperature compensated strain is denoted by $\mu$. The temperature compensated strain is a function of the SOC. That function is denoted by $h$. We determine $h$ empirically based on experimental data collected under standard static charge and discharge profiles.

The first equation essentially states that the SOC change is driven by the input current. The second equation states that the parameter $K$ is constant over time. The third equation states that observed strain is a function of SOC. To account for non-ideal behavior, we add noise $w_{q}$ and $w_{K}$ to the equations describing the dynamics of $q$ and $K$, as well as noise to the measurement equation for $\mu$.

To compute the function $h$, we subjected the module to static charge and discharge cycles as shown in Figure 6. Using the static cycles, the temperature compensation factor is first determined. We implemented an Extended Kalman filter (EKF) [22] to estimate the SOC during the US06 cycles based on the process and measurement equations described earlier. The states 
include $q$ and $K$. The process equation is linear in the states. The measurement equation though is nonlinear. Hence the need for an EKF.

The SOC is estimated as the average of the recovered FBG strain signal-based estimate and the Coulomb-count estimate (the latter being from the current signal). This is done since the pure FBG-based SOC estimation is challenged to some extent in the central flat region of wavelength shift vs. SOC curve, as shown in Figure 5 (b). The averaging is implemented as follows. At any given time instant, two estimates of $q$ and $K$ are obtained. The first set of estimates, $\widehat{q}_{1}$ and $\widehat{K}_{1}$, are obtained using a small value of $v_{\mu}$. This essentially implies that we trust the optical measurements.
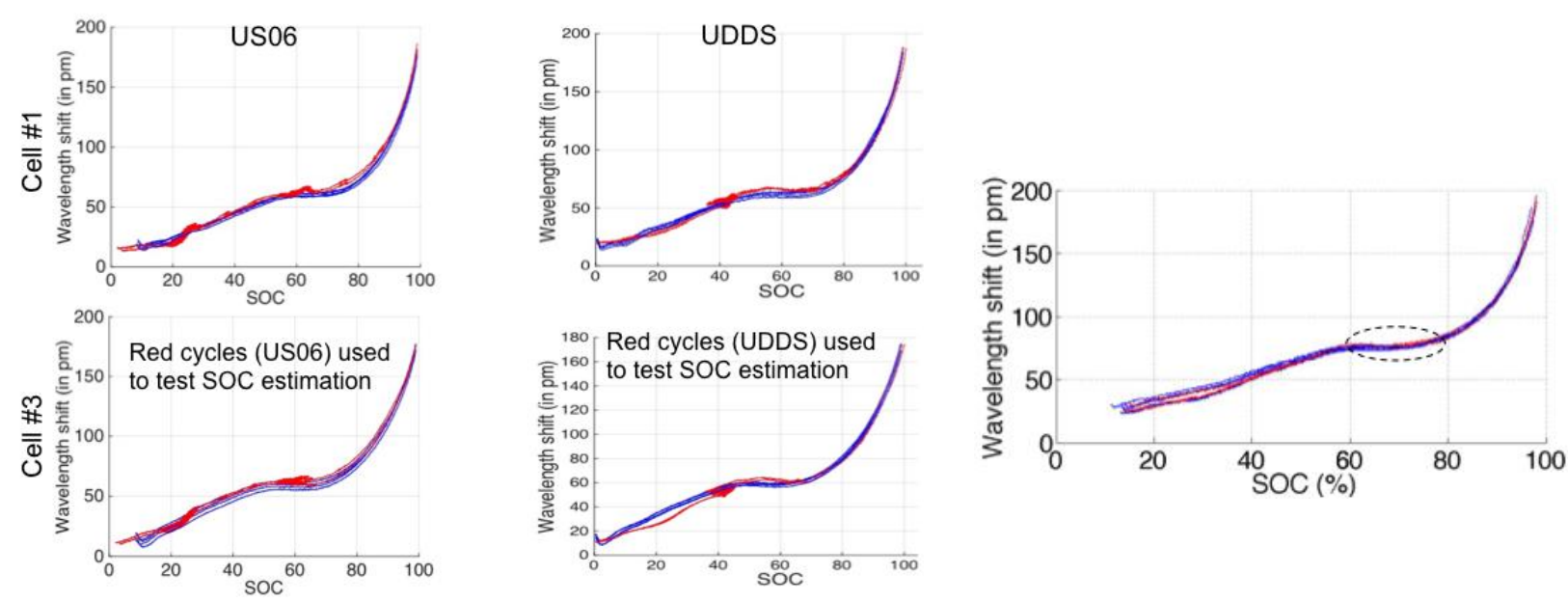

(a)

(b)

Figure 5: (a) Recovered internal strain signals from cells in 4-cell module for xEV dynamic cycles (in red), overlaid on static cycle curves (in blue), (b) central portion of FBG strain curve over which pure FBG-signal based SOC estimation is challenged. 
The second set of estimates, $\hat{q}_{2}$ and $\widehat{K}_{2}$, are obtained using a relatively larger value of $v_{\mu}$. This, on the other hand, implies low confidence in the optical measurements and, consequently, more emphasis on the prediction step of the EKF. Recall that the prediction step of the EKF [22] is essentially just Coulomb-counting. The two estimates are then combined heuristically as follows:

$$
\begin{gathered}
\widehat{[q} \quad \widehat{K}]=\Sigma_{2}\left(\Sigma_{1}+\Sigma_{2}\right)^{-1}\left[\begin{array}{ll}
\hat{q}_{1} & \widehat{K}_{1}
\end{array}\right]^{T}+\Sigma_{1}\left(\Sigma_{1}+\Sigma_{2}\right)^{-1}\left[\begin{array}{ll}
\hat{q}_{2} & \widehat{K}_{2}
\end{array}\right]^{T} \\
\Sigma=\left(\Sigma_{1}^{-1}+\Sigma_{2}^{-1}\right)^{-1}
\end{gathered}
$$

where $\Sigma_{1}$ and $\Sigma_{2}$ are the error covariances associated with the estimates $\left(\widehat{q}_{1}, \widehat{K}_{1}\right)$ and $\left(\hat{q}_{2}, \widehat{K}_{2}\right)$, respectively.

The SOC estimation results are summarized in Table 2 through Table 5. The tables show the mean percentage errors in SOC estimation for the cases when initial SOC estimation errors are $1 \%$ and $5 \%$. In each case, we compare the errors obtained via Coulomb Counting (CC) and our approach $(\mathrm{FO}+\mathrm{CC})$. Note that because $\mathrm{CC}$ is also our method to compute ground truth, the mean error in all $\mathrm{CC}$ cases remains identical to the initial SOC estimate error. As can be seen that when the initial error is $1 \%$, the mean SOC estimation error based on our approach $(\mathrm{FO}+\mathrm{CC})$ is well below 2.5\%, and the worst-case SOC accuracy is below 2.5\% for the xEV-relevant 5-95\% SOC window; see Figure 6. Further improvements are possible near the lower SOC values, where the errors increase due to differences in training and testing data. These can help further push the envelope for xEV battery utilization further beyond the 5\% and 95\% SOC limits, which are partly challenged today by SOC estimation accuracy. When the initial SOC estimate error is $5 \%$, the $\mathrm{CC}$ errors remain at $5 \%$ whereas the $\mathrm{FO}+\mathrm{CC}$ errors are $3 \%$ or lower with most of the cases being under $2.5 \%$. This further shows that the FO+CC approach is a more robust one and can tolerate large errors in initial estimate. 

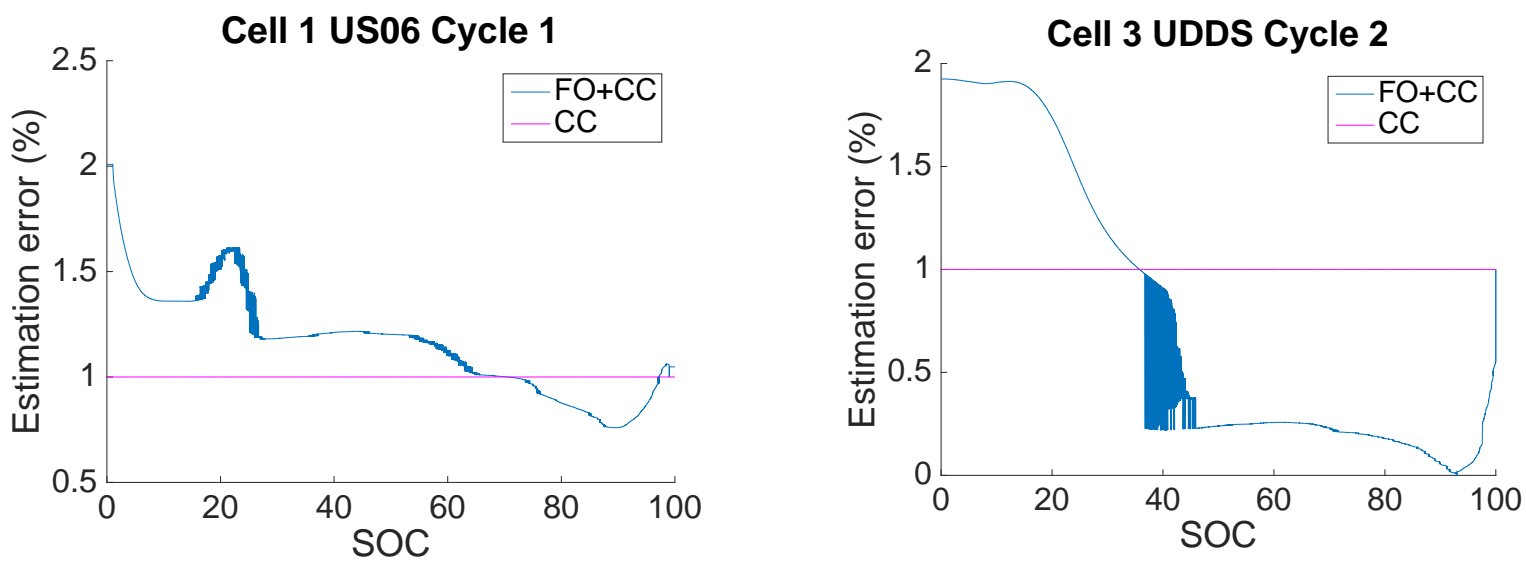

Figure 6: Plots of estimated SOC versus true SOC for an example US06 and a UDDS cycle showing larger errors at lower SOCs.

While these represent very promising initial results, the following points will need to be revisited to improve SOC estimation further:

a. Ground-truth SOC estimation is still a challenge. Currently this is obtained via Coulomb counting. It is unclear whether this is best ground-truth for dynamic cycles. Furthermore, the cells in the module are connected in parallel. Our initial setup here did not allow for measurement of individual currents in each cell.

b. It should also be noted the FBG base wavelength at start of discharge varies a bit cycle-cycle. This is a result of the FBG signal being sensitive to cell aging, as will be explored further in the next section. All the same, we used the same base wavelength across the cycles to be consistent. Eventually though, the SOC and $\mathrm{SOH}$ algorithms need to function in a closed loop where the SOC algorithm accounts for the changing cell capacity.

c. Some estimation filter parameter fine-tuning is needed. More uniformity in temperature compensation, ground truth estimation should obviate this need. 
d. The dynamic cycle hold times were limited to $1 \mathrm{~s}$ here; when attempting faster cycles with 0.1 s-hold times, we ran into cycler/amplifier hardware limitations.

\begin{tabular}{|c|c|c|}
\hline & FO + CC & CC \\
\hline Cell 1 US06 Cycle 1 & $1.16 \%$ & $1 \%$ \\
\hline Cell 1 US06 Cycle 2 & $0.76 \%$ & $1 \%$ \\
\hline Cell 3 US06 Cycle 1 & $1.18 \%$ & $1 \%$ \\
\hline Cell 3 US06 Cycle 2 & $0.49 \%$ & $1 \%$ \\
\hline
\end{tabular}

Table 2: SOC estimation results for US06 cycles with 4-cell module (internal sensing) and initial SOC error at 1 percent

\begin{tabular}{|c|c|c|}
\hline & FO + CC & CC \\
\hline Cell 1 US06 Cycle 1 & $3 \%$ & $5 \%$ \\
\hline Cell 1 US06 Cycle 2 & $2.6 \%$ & $5 \%$ \\
\hline Cell 3 US06 Cycle 1 & $3.07 \%$ & $5 \%$ \\
\hline Cell 3 US06 Cycle 2 & $2.37 \%$ & $5 \%$ \\
\hline
\end{tabular}

Table 3: SOC estimation results for US06 cycles with 4-cell module (internal sensing) and initial SOC error at 5 percent 


\section{Impact of Cell Aging and SOH Estimation}

Following the SOC estimation tests on the 4-cell module, aggressive charging/discharging was done on that module (with all 4 cells connected electrically in parallel) over 100 cycles to induce degradation and test the feasibility of using the internal FBG sensor signals for high-accuracy SOH estimation and capacity degradation prediction. The current profile for this series of aggressive tests consists of charging at $\mathrm{C}$ rates followed by discharging corresponding to the US06 drive profile.

A clear correlation between the FBG wavelength at the end of the charge cycle and the charge capacity was observed, as shown in Figure 7 (a). This suggested that this feature would be useful to predict capacity and failure (20\% capacity drop) ahead a few cycles. Accordingly, a linear Kalman filtering algorithm was used to predict capacity. The first 40 cycles from cell \#3 in the module was used as training set while the other cycles from that cell and cell \#1 were used as representative test sets to validate the algorithm. The $\mathrm{SOH}$ (capacity prediction) algorithm is summarized here:

$$
\begin{gathered}
\mathrm{C}(\mathrm{k}+1)=\mathrm{C}(\mathrm{k})+\mathrm{Q}(\mathrm{k}) \Delta T \\
\mathrm{Q}(\mathrm{k}+1)=\mathrm{Q}(\mathrm{k}) \\
\mu_{\max }(\mathrm{k})=\mathrm{B}_{0}+\mathrm{B}_{1} \cdot \mathrm{C}(\mathrm{k})+\mathrm{B}_{2} \cdot \mathrm{Q}(\mathrm{k})
\end{gathered}
$$

Where:

- $\mathrm{k}$ denotes cycle number

- $\mathrm{C}(\mathrm{k})$ denotes the capacity at cycle $\mathrm{k}$

- $\mathrm{Q}(\mathrm{k})$ denotes the rate of change of capacity at cycle $\mathrm{k}$

- $\quad \mu_{\max }(\mathrm{k})$ denotes the maximum strain at the end of charge for cycle $\mathrm{k}$

- $\mathrm{B}_{0}, \mathrm{~B}_{1}, \mathrm{~B}_{2}$ - determined using training data (first 40 cycles of cell \#3) 
Importantly, the algorithm did not utilize the electrical signals beyond their use as ground truth for the training cycles. The prediction results from this algorithm are shown in Figure 7 (b) and (c), and summarized in Table 6. There is a slight jump in pattern after cycle \#26, which is attributable to a changed light source with slightly different spectrum in our setup causing some wavelength shifts at the end of that cycle. Our algorithm focused on prediction beyond this initial window. As seen in Table 6, the model was able to achieve $1.1 \%$ or better accuracy with a 5cycle prediction window over the $70+$ test cycles. Thus, the model is expected to predict cell end-of-life failure (i.e., 20\% capacity drop) with high accuracy over a 3-5 cycle prediction horizon.

\section{Conclusions and Future Directions}

In this two-part paper, we presented a promising new embedded sensing option for internal cell monitoring, fiber-optic sensors. In the companion first paper, we described the process of fabrication of high-performance, xEV-grade large-format Lithium-ion pouch cells with embedded fiber-optic (FO) sensors attached to the electrode. The seal integrity, capacity retention, and projected cycle life are all comparable to contemporary xEV cells without embedded sensors. The cells can be integrated into existing xEV module designs. The massvolume cost estimates for the FO sensing system are in the range of a few hundreds of U.S. dollars and are thus comparable and competitive with that of electrical sensing for $\mathrm{xEV}$ BMS today. In this second part, we focused on using the measurements obtained from the embedded sensors for SOC and SOH estimation. We showed that the strain measured using FO sensors can be used to estimate SOC with less than $2.5 \%$ errors under different temperature conditions and under dynamic cycling. We also presented encouraging results from our initial tests showing that strain can also be used to predict the cell capacity up to 10 cycles ahead with approximately $2 \%$ 
or lower errors. More rigorous testing over a broader set of validation scenarios in larger modules is currently being performed in partnership with an xEV original equipment manufacturer. In addition, we are also exploring the use of FO strain signals to estimate the stateof-power (SOP) which is another key function of current battery management systems.

\begin{tabular}{|c|c|c|}
\hline & FO + CC & CC \\
\hline Cell 1 UDDS Cycle 1 & $0.34 \%$ & $1 \%$ \\
\hline Cell 1 UDDS Cycle 2 & $0.45 \%$ & $1 \%$ \\
\hline Cell 3 UDDS Cycle 1 & $0.55 \%$ & $1 \%$ \\
\hline Cell 3 UDDS Cycle 2 & $0.75 \%$ & $1 \%$ \\
\hline
\end{tabular}

Table 4: SOC estimation results for UDDS cycles with 4-cell module (internal sensing) and initial SOC error at 1 percent

\begin{tabular}{|c|c|c|}
\hline & FO + CC & CC \\
\hline Cell 1 UDDS Cycle 1 & $2.03 \%$ & $5 \%$ \\
\hline Cell 1 UDDS Cycle 2 & $1.29 \%$ & $5 \%$ \\
\hline Cell 3 UDDS Cycle 1 & $1.69 \%$ & $5 \%$ \\
\hline Cell 3 UDDS Cycle 2 & $1.13 \%$ & $5 \%$ \\
\hline
\end{tabular}

Table 5: SOC estimation results for UDDS cycles with 4-cell module (internal sensing) and initial SOC error at 5 percent 


\begin{tabular}{|l|l|l|}
\hline & Mean error & Max error \\
\hline Predict 1 cycle ahead & $0.14 \%$ & $0.38 \%$ \\
\hline Predict 5 cycles ahead & $0.24 \%$ & $1.1 \%$ \\
\hline Predict 10 cycles ahead & $0.4 \%$ & $2.2 \%$
\end{tabular}

Table 6: Prediction errors for cell \#1 (cycle \#26 onwards) using model built on $1^{\text {st }} 40$ cycles from cell \#3

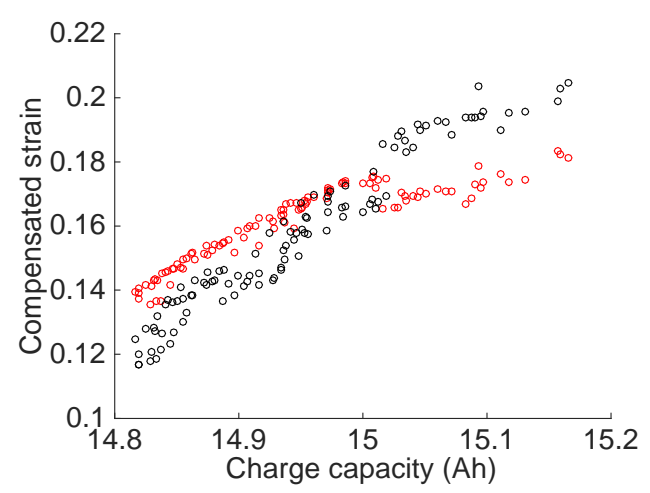

(a)

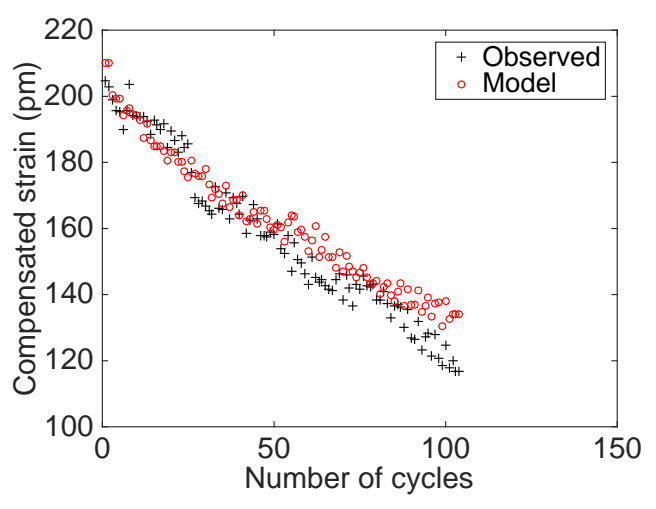

(b)

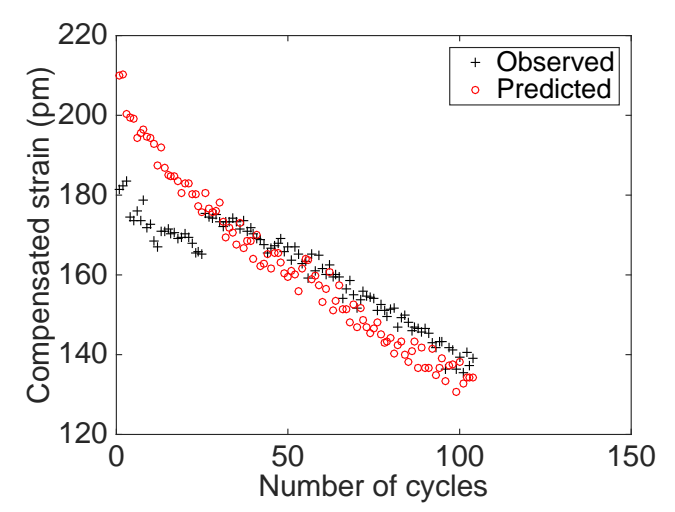

(c)

Figure 7: (a) Plot showing correlation between FBG recovered strain at the end of charge and charge capacity (which decreased monotonically with increasing cycle number) in the aggressive cycle tests (red circles: cell \#1, black circles: cell \#3). Model predictions and observed capacity over the 100+ aggressive cycles for (b) Cell \#3, and (c) Cell \#1 


\section{Acknowledgements}

The information, data, or work presented herein was funded in part by the Advanced Research Projects Agency-Energy (ARPA-E), U.S. Department of Energy, under Award Number DE-AR0000274.

\section{References}

[1] T. Turrentine, Plug-in Hybrid Electric Vehicle Research Roadmap. UC Davis Plug-In Hybrid Electric Vehicle Research Center, June 2011.

[2] K. A. Smith, C.-Y. Wang, Power and thermal characterization of a lithium-ion battery pack for hybrid-electric vehicles. J. Power Sources, 160, 662-673, 2006.

[3] N. A. Chaturvedi, R. Klein, J. Christensen, J. Ahmed, A. Kojic, Algorithms for advanced battery-management systems. Control Systems, IEEE, 30(3), 49-68, 2010.

[4] K. S. Ng, C.-S. Moo, Y.-P. Chen, and Y.-C. Hsieh, "Enhanced coulomb counting method for estimating state-of-charge and state-of-health of lithium-ion batteries," Applied energy 86, no. 9: 1506-1511, 2009.

[5] S. Lee, J. Kim, J. Lee, and B. H. Cho, "State-of-charge and capacity estimation of lithium-ion battery using a new open-circuit voltage versus state-of-charge," Journal of Power Sources, 185, no. 2: 1367-1373, 2008.

[6] S. Piller, M. Perrin and A. Jossen, "Methods for state-of-charge determination and their applications", Journal of power sources, Vol. 96, No. 1, pp.113-120, 2001.

[7] Y. Chiang, S. Wu-Yang and K. Jia-Cheng, "Online estimation of internal resistance and open-circuit voltage of lithium-ion batteries in electric vehicles." Journal of Power 
Sources 196, no. 8 (2011): 3921-3932.

[8] S. Buller, M. Thele, E. Karden, and R. W. De Doncker, "Impedance-based non-linear dynamic battery modeling for automotive applications," Journal of Power Sources 113, no. 2: 422-430, 2003.

[9] H. He, R. Xiong, and J. Fan, "Evaluation of lithium-ion battery equivalent circuit models for state of charge estimation by an experimental approach," Energies 4, no. 4: 582-598, 2011.

[10] J. Lee, O. Nam, and B. H. Cho, "Li-ion battery SOC estimation method based on the reduced order extended Kalman filtering," Journal of Power Sources 174, no. 1: 9-15, 2007.

[11] S. Santhanagopalan, Q. Guo, P. Ramadass, and R. E. White, "Review of models for predicting the cycling performance of lithium ion batteries," Journal of Power Sources 156, no. 2: 620-628, 2006.

[12] S. J. Moura, N. A. Chaturvedi, and M. Krstic, "PDE estimation techniques for advanced battery management systems-Part I: SOC estimation,” In American Control Conference (ACC), 2012, pp. 559-565. IEEE, 2012.

[13] Y.-J. Rao, “In-fibre Bragg grating sensors,” Meas. Sci. Technology, Bd. 8, pp. 355-375, 1997

[14] R. Van Steenkiste. Strain and temperature measurement with fiber optic sensors. Technomic Publishing, PA, 1997.

[15] N. Zhang, H. Tang, Dissecting anode swelling in commercial lithium-ion batteries, Journal of Power Sources, Bd. 218, pp. 52-55, 2012. 
[16] J. H. Lee, H. M. Lee, S. Ahn, Battery dimensional changes occurring during charge/discharge cycles - thin rectangular lithium ion and polymer cells, Journal of Power Sources, Bd. 1, 2119-121, pp. 833-837, 2003.

[17] M. Hahn, H. Buqa, P. W. Ruch, D. Goers, M. E. Spahr, J. Ufheil,P. Novak, R. Koetz, A Dilatometric Study of Lithium Intercalation into Powder-Type Graphite Electrodes, Electrochemical and Solid-State Letters, Bd. 11, Nr. 9, pp. A151-A154, 2008.

[18] L.W. Sommer, A. Raghavan, P. Kiesel, B. Saha, J. Schwartz, A. Lochbaum, A. Ganguli, C.-J. Bae, and M. Alamgir, "Monitoring of Intercalation Stages in Lithium-Ion Cells over Charge-Discharge Cycles with Fiber Optic Sensors," Journal of The Electrochemical Society, 162(14), A2664-A2669, 2015.

[19] D. J. Berndt and James Clifford, Using Dynamic Time Warping to Find Patterns in Time Series, KDD workshop, Vol. 10, No. 16, 1994.

[20] United States Environmental Protection Agency (EPA) US06 or Supplemental Federal Test Procedure (SFTP). < https://www.epa.gov/emission-standards-reference-guide/epaus06-or-supplemental-federal-test-procedure-sftp >; [cited April 18, 2016]

[21] The United States Environmental Protection Agency (EPA) Urban Dynamometer Driving Schedule (UDDS), <https://www.epa.gov/emission-standards-reference-guide/epa-urbandynamometer-driving-schedule-udds>; [cited April 18, 2016]

[22] B D. O. Anderson and J. B. Moore. Optimal filtering. Courier Corporation, 2012. 